Vol. 6, Num. 1, 2021

\title{
ACQUISITION AND PROCESSING OF DATA IN CPS FOR REMOTE MONITORING OF THE HUMAN FUNCTIONAL STATE
}

\author{
Petro Hupalo and Anatoliy Melnyk \\ Lviv Polytechnic National University, 12, Bandera Str, Lviv, 79013, Ukraine \\ Author's e-mail:gypalo911@gmail.com; anatoliy.o.melnyk@lpnu.ua
}

https://doi.org/10.23939/acps2021.01.

Submitted on 02.05.2021

(C) Hupalo P., Melnyk A., 2021

\begin{abstract}
Data acquisition and processing in cyber-physical system for remote monitoring of the human functional state have been considered in the paper. The data processing steps, strategies for multi-step forecasting evaluation metrics and machine learning algorithms to be implemented have been analysed and described. What is important, this way it will be possible to track the condition of the sick and response to the health changes in advance.
\end{abstract}

Index terms: biometric data, data collection, machine learning, neural networks, remote monitoring of human state.

\section{INTRODUCTION}

Wearable technology has added a whole new dimension in the healthcare system by real-time continuous monitoring of human body physiology. They are used in daily activities and fitness monitoring and have even penetrated in monitoring the health condition of patients suffering from chronic illnesses.

The wearable medical devices face the challenges of reliability, accuracy, precision, and robustness. There are two important sub-sections for a complete wearable medical device: hardware, which involves the sensor selection and characterization, noise removal from the acquired signals, and communication to the decision-making subsystem and software, which involves taking decisions based on the acquired signals. The second subsystem is where the machine learning comes into play, and the recent outburst of development in the field of machine learning has increased the possibility of remote monitoring and diagnosing with the acquired data from the wearable devices.

The machine learning block of the wearable devices involves various steps such as pre-processing, feature selection, training on the labelled dataset, and testing to verify its accuracy and competency. Thus, it is inevitable that machine learning methods that include algorithm training are required to enable the diagnosis of different diseases [1].

Human state remote monitoring tools contain wireless wearable devices that measure its status parameters, cloud storage and data processing services. The work of remote monitoring tools consists in the transfer of data from wireless wearable devices to cloud services and the interaction of these services with mobile user terminals [2].

This paper is intended to describe the system of remote monitoring of the human functional state and give an overview of the implementation of machine learning for healthcare diagnostics using data collected with wireless wearable devices.

\section{CURRENT STATUS OF THE PROBLEM}

With the popularization of smartphone use, applications of wearable technology have exploded, converged with artificial intelligence (AI), IoT, and smart sensing. Today, they are widely used not only in the healthcare industry but also in gaming, communications, industrial operations, and safety. For example, in healthcare, IBM offers a wide variety of mobile services through its Mobile Wireless Health Solutions, and GE Healthcare has developed Vscan to provide more accurate and faster healthcare services that are not constrained by time and place.

Wearable devices in healthcare consist of various devices, including wearable fitness trackers, smart health watches, wearable ECG monitors, wearable blood pressure monitors, and biosensors. The development of smartphone apps has helped provide a wide range of healthcare services and this trend is expected to accelerate in the future. Apple's Watch, Garmin's series of devices, Galaxy Watch of Samsung Electronics, Fitbit's, and Xiaomi's Mi Band are good examples [3].

Developed data acquisition and processing tools consist of the next parts:

- wearable devices (Garmin, Apple Watch, any other that can export data to health data aggregators like Apple Health)

- mobile application (both for iOS and Android)

- $\quad$ server-side (to collect and process aggregated data)

- machine learning models (that are training, testing and doing prediction on the server)

Based on that structure a contemporary and convenient system for people who want to analyze and share their functional state data to their doctor was created. Using that system for 3 months by 10 regular users, a dataset of their functional state mostly continuously every day was collected. Among indicators that had been collected there were oxygen saturation $(\mathrm{SpO} 2)$, heart rate and respiration rate with the further growth of wearable devices that would be possible to extend that list with such important indicators as temperature, glucose level and blood pressure. Each additional sensor included in a device can be used to add additional types of metrics for the user or supply internal algorithms with additional data to improve accuracy of 
already available metric types. However, additional sensors affect price and power consumption [4].

If to look from the side of how much data is being collected using wearable devices and their ability to obtain large amounts of information in real time, it is obvious to say that data has become too big in amount, and it changes shape almost as fast as it accumulates. Big data has emerged as the catch-all term for both the data itself and also for the tools and practices people use to get it under control. That is why, that is a good field for machine learning problems of forecasting, classification and clustering [3].

Consequently, the main aim of the data acquisition and processing tools for remote monitoring of the human functional state is not only to process and share data to the doctors, but also to give them advanced insight information to define and prevent many illnesses on early stage. Especially during the Covid-19 pandemic when any minor changes in the main human health indicators can have their effects very quickly. That is why, the reaction on that should not be delayed.

\section{DATA ACQUISITION IN CYBER-PHYSICAL SYSTEM FOR REMOTE MONITORING OF THE HUMAN FUNCTIONAL STATE}

\section{A. SELECTION OF WEARABLES}

New fitness trackers and smartwatches are released to the consumer market every year. These devices are equipped with different sensors, algorithms, and accompanying mobile apps. With recent advances in mobile sensor technology, privately collected physical activity data can be used as an addition to existing methods for health data acquisition in research. Furthermore, data collected from these devices have possible applications in patient diagnostics and treatment. With an increasing number of diverse brands, there is a need for an overview of device sensor support, as well as device applicability in research projects[5] [5].

The main goal was to define devices that can be used to track lungs health state due to the Covid-19 pandemic. Beside smart watches there were also analyzed different pulse-oximeters which could satisfy defined characteristics.

Pulse oximeters, which are used in cyber-physical systems for 24/7 monitoring of lung function, must be connected to a smartphone via the Bluetooth interface to transmit measurement data.

Mobile applications connected to heart rate monitors must integrate with GoogleFit on Android and HealthKit on iOS to combine data from different sensors.

Pulse oximeters must be portable and have a long battery life.

The accuracy of their blood oxygen saturation measurement should not be lower than in calibrated medical devices.

Pulse oximeters should measure blood oxygen saturation with $\mathrm{SpO} 2$ over certain time intervals without the human intervention.

Based on the above, we can form the following criteria for comparison and selection of pulse oximeters [6]:

- Price

- Portability
- $\quad$ Size and weight

- Wireless communication function with a smartphone (via Bluetooth)

- Ability to export blood oxygen saturation data to GoogleFit on Android and HealthKit on iOS

- Measurement accuracy

- Duration of autonomous work

- Periodic measurement.

Based on the conducted analysis of pulse oximeters available on the market, there were selected devices that can be used in cyber-physical systems for $24 / 7$ monitoring of lung functions, what means they are portable, have wireless communication function with a smartphone (via Bluetooth) and provide the ability to export saturation data blood oxygen $\mathrm{SpO} 2$ to GoogleFit on Android and HealthKit on iOS. Here are some specific devices that were used by people who exported data to the created system:

- Garmin Vivoactive 4/4s, Venu

- Garmin Fenix 6/5

- Garmin Vivomove 4

- $\quad$ Apple Watch Series 6

- Huawei Band 4

- Beurer PO 60 Bluetooth ${ }^{\circledR}$ pulse oximeter

\section{B. DATA TRANSFERRING}

As it was mentioned above, it is important to choose devices that can measure health indicators continuously and export data via Bluetooth or WiFi.

The measuring device includes sensors that collect information and transmit it to a mobile terminal.

The mobile terminal includes a block for receiving data from measuring device sensors, a data transfer unit to the cloud service (for interaction with the cloud service), an authentication unit (for interaction with the authentication service) and a secure storage (for storing the necessary personal data).

The cloud service receives data from the mobile terminal (using a secure https channel) as a REST API. As in the mobile terminal, there is an authentication unit. Fig.1 shows the transmission process from the measuring device to mobile terminal and cloud service.

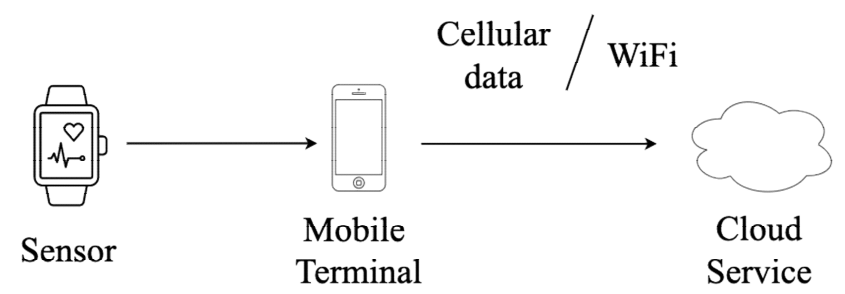

\section{Fig. 1. Transmission of data measured with a measuring device using Bluetooth technology to the mobile terminal and cloud service.}

The information measured by the sensor is transmitted using Bluetooth technology to the user mobile terminal. Then the data are automatically sent through the network of the mobile operator or WiFi to the server without the user's participation. The only thing user should care about is that his mobile terminal should be connected to the internet. 


\section{DATA ACQUISITION AFTER TRANSFERRING OR AGGREGATION FROM EXTERNAL SOURCES}

After the data from devices was accepted by Cloud Service it needs to be properly stored in the database. When creating systems that collect data from different devices or services that is necessary to process it differently depending on the technologies that are provided by the device manufacturer.

Here is presented small part of response from Garmin health API after user synchronized his devices with the Cloud Service:

$\{"$ dailies $"=>$ [ $\{$ "userId"=>"02236a89-0fb8-4a2e-9f6a4a9b6b84e825",

"summaryId"=>"x35eb9dc-60ac13d0-d728-6",

"calendarDate"=>"2021-05-25",

"activityType"=>"WALKING",

"activeKilocalories" $=>85$,

"bmrKilocalories" $=>1390$,

"steps" =>5481,

"distanceInMeters" $=>4563.0$,

"durationInSeconds" $=>55080$,

"activeTimeInSeconds"=>4737,

"startTimeInSeconds" $=>1621890000$,

"startTimeOffsetInSeconds"=>10800,

"moderateIntensityDurationInSeconds" $=>0$,

"vigorousIntensityDurationInSeconds" $=>0$,

"floorsClimbed" $=>1$,

"minHeartRateInBeatsPerMinute" $=>38$,

"maxHeartRateInBeatsPerMinute" =>103,

"averageHeartRateInBeatsPerMinute" $=>55$,

"restingHeartRateInBeatsPerMinute" $=>40$,

"timeOffsetHeartRateSamples $"=>\{" 15 "=>58, \quad " 30 "=>58$,

$" 45^{\prime \prime}=>58, " 60 "=>58, " 75 "=>58, " 90 "=>58, " 105 "=>58$,

$" 120 "=>58, \quad " 135^{\prime \prime}=>58, \quad " 150 "=>58, \quad " 165^{\prime \prime}=>58$,

$" 180 "=>58, \quad " 195 "=>59, \quad " 210 "=>59, \quad " 225 "=>59$,

"240"=>59, "255"=>58, "270"=>58, "285"=>58,

$" 300 "=>58, \quad " 315 "=>59, \quad " 330 "=>59, \quad " 345 "=>59$,

"360"=>59, "375"=>61, "390"=>61,, ... J\}

It does not look pretty good to be understood for the ordinary user. And furthermore, all this data has no need to be stored on the web-server. That is why the data should be processed to a better presentable form to store and then retrieve all these time series.

\section{IV.DATA PROCESSING AND FORECASTING USING MACHINE LEARNING IN CYBER-PHYSICAL SYSTEM FOR REMOTE MONITORING OF THE HUMAN FUNCTIONAL STATE}

\section{DATA PROCESSING}

During the COVID-19 pandemic, due to the large number of patients in hospitals, doctors do not have enough time to be distracted to self-execute the analysis of indicators of various medical measuring devices. It is very important that the information is presented in a processed form. In this case, from the point of view of clarity, a graphical representation of information is the best for perception.
Developed system presents graphs of average values of indicators for the day, week and month, as well as graphs of minimum and maximum values. For example, in Fig. 2 the graphs of average (top), minimum and maximum (bottom) values of blood oxygen saturation of a patient are shown (obtained during the week). As it can be seen, the average values are within acceptable limits. However, the minimum values of the first patient are a cause for concern. Therefore, the doctor should pay attention to them and conduct additional research, in particular to analyze all the indicators obtained from the first patient. To do this, in the application it is possible to view all the measured indicators on the basis of which the graphs are based. The indicators of the second patient are satisfactory.

In the process of measurement, the question of reliability of the measured indicators is important. In particular, the accuracy of the measurement may be affected by external factors, such as the position of the human body or the movement during the measurements. For example, measuring blood oxygen saturation while a patient is moving may be inaccurate. Therefore, it is necessary to process the received indicators. That is considered inappropriate to use tracking filters, in particular the Kalman filter, because they distort the obtained indicators. At the same time, creating a histogram of indicators for the period of time and the ratio of the periods of maximum or minimum values of the indicators to the total measurement time will be useful.

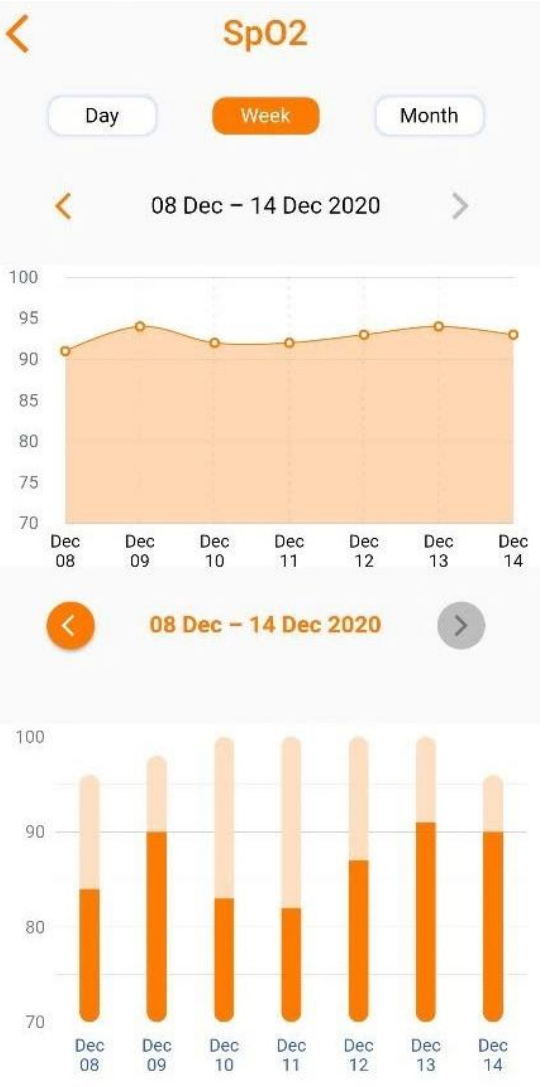

Fig. 2. Presentation of a patient data in the mobile application.

All the collected data is a time series. A time series is a sequence $S$ of historical measurements $y_{t}$ of an observable 
variable $y$ at equal time intervals. That means that one measurement corresponding to concrete time cannot be repeated. As it was described earlier, wearable devices are measuring simultaneously 3 main parameters: Oxygen Saturation, Pulse and Respiration Rate. With the collected data and a goal to predict lungs state for some period in the future there is a need of implementation machine learning algorithms.

\section{E. MACHINE LEARNING STRATEGIES FOR TIME SERIES FORECASTING}

Time series are studied for several purposes such as the forecasting of the future based on knowledge of the past, the understanding of the phenomenon underlying the measures, or simply a succinct description of the salient features of the series. Forecasting future values of observed time series plays an important role in nearly all fields of science and engineering, such as economics, finance, business intelligence, meteorology and telecommunication. An important aspect of the forecasting task is represented by the size of the horizon. If one-step forecasting of a time series is already a challenging task, performing multi-step forecasting is more difficult because of additional complications, like accumulation of errors, reduced accuracy, and increased uncertainty [7].

There are many things to keep in mind when a new time series prediction problem arises. The choice made at the beginning directly affects each step of the project. You can very quickly narrow down the options by doing a number of basic questions about a specific problem of time series forecasting. By considering and answering questions independently within each topic, the type of problem is narrowed and the choice of algorithms for the project is simplified.

Based on data from different types of pulse oximeters, the aim was to analyze how to create a multi-stage forecasting system that can predict some important indicators of human health for different periods of time ahead.

After a brief study and analysis of a series of available data, it was concluded that the studied data type, namely unstructured time series with more than one parameter, measured simultaneously in time. Working with this data involves forecasting $\mathrm{n}$-steps forward and the forecasting model must learn from the new data before each prediction.

Target users are people who have or have had COVID19 or have symptoms of other respiratory illnesses.

To implement the ideas described above, the overall structure of the system will have the following steps:

- Data collection

- Preparation and pre-processing of data

- Choice of algorithms and testing

- Comparison of algorithm results and selection of optimal ones

- Data visualization

First of all, the main issue after data acquisition is to prepare them properly so that they match the algorithm. There is one type of data preparation for controlled machine learning models, and quite another to prepare data for neural network connections - CNN or LSTM.
Traditionally, time series prediction is dominated by linear algorithms such as ARIMA, as they are well understood and effective to solve many problems. But these classic methods also have a number of limitations, such as:

- Completeness of data: missing data or damaged data will usually make the forecast unreliable

- One-dimensional data: Many real-world problems have several input variables.

- One-Step Forecasts: Many real-world problems require long-term forecasts.

Machine learning techniques can be effective for more complex time series prediction problems with multiple input variables, complex nonlinear relationships, and missing data. For efficient operation, these methods often require manually developed functions [8].

\section{F. DATASET}

An unstructured data is the information that does not adhere to a pre-defined model or organizational framework. In the healthcare sector, it could be materialized in terms of better management, care and low-cost treatments. We are miles away from realizing the benefits of big data in a meaningful way and harnessing the insights that come from it. In order to achieve these goals, we need to manage and analyze the big data in a systematic manner [9][9].

It was described above that the collected data are dynamic multidimensional time series with n-steps forward prediction, which means that the algorithmic model will be based on new data before each prediction and has several input variables measured over time.

Fig. 3 shows the collected data of one person during the period of 2 months. Mainly, indicator values were measured for every minute, except periods of activity or when device was unused. That is a typical example of one-dimensional time series.

\begin{tabular}{|c|c|c|}
\hline & date & spo2 2 \\
\hline 0 & $2020-12-1300: 04: 00$ & 99 \\
\hline 1 & $2020-12-13$ 00:05:00 & 97 \\
\hline 2 & $2020-12-1300: 06: 00$ & 96 \\
\hline 3 & $2020-12-13$ 00:07:00 & 95 \\
\hline 4 & $2020-12-13$ 00:08:00 & 95 \\
\hline ... & ... & .. \\
\hline 28918 & $2021-02-09$ 16:53:00 & $9 \mathrm{~s}$ \\
\hline 28919 & $2021-02-09$ 16:54:00 & 99 \\
\hline 28920 & $2021-02-09$ 16:55:00 & 99 \\
\hline 28921 & 2021-02-09 16:56:00 & 98 \\
\hline 28922 & 021-02-09 16:57:00 & 98 \\
\hline
\end{tabular}

Fig.3. Example of collected SpO2 time series data for one person

The number of observations recorded over time in the time series data set matters. Traditionally, different names are used:

- One-dimensional time series: these are data sets where only one variable is observed at a time, such as temperature per hour. 
- Multidimensional time series: these are data sets where two or more variables are observed simultaneously.

Most methods of time series analysis, as well as books on the subject, focus on one-dimensional data. This is because they are the easiest to understand and work with. Multidimensional data is often more difficult to process. They are more difficult to model and often many classical methods do not show sufficient results.

In Fig. 4 there is an example of collected data from wearable devices with selected features based on the task of the oxygen saturation forecasting. Anthropometric data helps to distinguish time series of different groups of people.

\begin{tabular}{|c|c|c|c|c|c|c|c|c|c|}
\hline & & & & Sele & cted fea & tures & & & \\
\hline & height & weight & $\operatorname{sex}$ & age & spo2_o & spo2_1 & $\ldots$ & spo2_18 & spo2_19 \\
\hline 24 & 185 & 81 & 0 & 21 & 90 & 92 & $\ldots$ & 96 & 95 \\
\hline 25 & 185 & 81 & 0 & 21 & 92 & 92 & $\ldots$ & 95 & 95 \\
\hline 26 & 185 & 81 & 0 & 21 & 92 & 94 & $\ldots$ & 95 & 94 \\
\hline 27 & 185 & 81 & 0 & 21 & 94 & 94 & $\ldots$ & 94 & 93 \\
\hline 28 & 185 & 81 & 0 & 21 & 94 & 94 & $\ldots$ & 93 & 94 \\
\hline - & $\ldots$ & $\ldots$ & $\ldots$ & .. & $\ldots$ & $\ldots$ & ... & $\ldots$ & $\ldots$ \\
\hline 10194 & 179 & 75 & 1 & 26 & 91 & 92 & $\ldots$ & 98 & 98 \\
\hline 10195 & 179 & 75 & 1 & 26 & 92 & 92 & $\ldots$ & 98 & 89 \\
\hline 10196 & 179 & 75 & 1 & 26 & 92 & 92 & $\ldots$ & 89 & 88 \\
\hline 10197 & 179 & 75 & 1 & 26 & 92 & 92 & $\ldots$ & 88 & 92 \\
\hline 10198 & 179 & 75 & 1 & 26 & 92 & 91 & $\ldots$ & 92 & 91 \\
\hline
\end{tabular}

Fig.4. An example of a multidimensional time series for multi-step sequential prediction.

For these purposes, it is necessary to determine the algorithms that best suit them. To choose the most accurate model, one needs to conduct several experiments with the following algorithms:

- Autoregression.

- Linear and nonlinear machine learning.

- Ensemble machine learning.

- Deep learning.

DL approaches differ from shallow ML algorithms in terms of scalability, i.e., depth (number of hidden layers) and width (number of cells or units or neurons in each layer). DL (or deep ML) is a scalable ML approach capable of scaling to the data size in terms of high number of data samples or data dimensionality. DL is applied to artificial neural networks (ANN or NN) and that is why it is also known as deep neural networks (DNN).

Deep Learning is loosely inspired by the visual cortex. It is mimicking our brain in terms of learning and recognizing the spatial and temporal patterns (or spatiotemporal) in the data. DNN are basically deep hierarchical layers of perceptrons, as artificial neurons, for representation and regression learning [10][11].

Deep learning in healthcare provides doctors with the analysis of any disease accurately and helps them treat them better, thus resulting in better medical decisions. Deep learning technologies can be applied to hospital management information systems to achieve: lower cost, fewer hospital stays, and its length, control of insurance fraud, change detection in disease patterns, high-quality healthcare, and better efficiency of medical resource allocation [11]. One or more models that would be most accurate in the test data set would be included in the prediction system.

\section{G. EVALUATION METRICS}

Before forecasting, it should be clear how to measure the quality of the predictions. There are many metrics that can be used for this.

Time series prediction performance measures provide a summary of the skill and capability of the forecast model that made the predictions. There are many different performance measures to choose from. It can be confusing to know which measure to use and how to interpret the results. Here are the most common Forecasting Performance Measures [13]:

- $\quad$ Forecast Error (or Residual Forecast Error)

The forecast error is calculated as the expected value minus the predicted value. This is called the residual error of the prediction.

forecast error $=$ expected value - predicted value

- Mean Forecast Error (or Forecast Bias)

Mean forecast error is calculated as the average of the forecast error values.

mean forecast error $=$ mean (forecast error)

- Mean Absolute Error

The mean absolute error, or MAE, is calculated as the average of the forecast error values, where all of the forecast values are forced to be positive. Forcing values to be positive is called making them absolute.

mean absolute error $=$ mean (abs (forecast error))

- Mean Squared Error

Mean forecast error is calculated as the average of the forecast error values.

mean squared error $=$ mean (forecast error 2 )

- $\quad$ Root Mean Squared Error

The mean squared error described above is in the squared units of the predictions. It can be transformed back into the original units of the predictions by taking the square root of the mean squared error score.

$$
\text { rmse }=\sqrt{\text { mean_squared_error }}
$$

\section{H. MULTI-STEP FORECASTING STRATEGIES}

There can be considered three main strategies to show how learning techniques can be used to tackle the multi-step forecasting problem. Namely, there are the following strategies: recursive, direct and multiple output.

A multi-step time series forecasting task consists of predicting the next $\mathrm{H}$ values $\left[\mathrm{y}_{\mathrm{N}+1}, \ldots, \mathrm{y}_{\mathrm{N}+\mathrm{H}}\right]$ of a historical time series $\left[\mathrm{y}_{1}, \ldots, \mathrm{y}_{\mathrm{N}}\right]$ composed of $\mathrm{N}$ observations, where $\mathrm{H}$ $>1$ denotes the forecasting horizon [12].

The Recursive strategy trains first one-step model $f$ :

$y_{t+1}=f\left(y_{t}, \ldots, y_{t-n+1}\right)+w_{t+1}$, with $t \in\{n, \ldots, N-1\}$ and then uses it

recursively for returning a multi-step prediction. A wellknown drawback of the recursive method is its sensitivity to 
the estimation error, since estimated values, instead of actual ones, are more and more used when we get further in the future.

The Direct strategy learns independently $\mathrm{H}$ models $\mathrm{f}_{\mathrm{h}}$ :

$\mathrm{y}_{\mathrm{t}+\mathrm{h}}=\mathrm{f}_{\mathrm{h}}\left(\mathrm{y}_{\mathrm{t}}, \ldots, \mathrm{y}_{\mathrm{t}-\mathrm{n}+1}\right)+\mathrm{w}_{\mathrm{t}+\mathrm{h}}$, with $\mathrm{t} \in\{\mathrm{n}, \ldots, \mathrm{N}-\mathrm{H}\}$ and $\mathrm{h} \in\{1, \ldots, \mathrm{H}\}$

and returns a multi-step forecast by concatenating the $\mathrm{H}$ predictions.

A detailed review and comparison of strategies for multi-step time series forecasting based on the local learning algorithm is presented in [8].

\section{MODELS TRAINING AND EXPERIMENTAL RESULTS}

The process of choosing a model can usually be divided into the following stages [13]:

1) The division of the data set into training and test sets.

2) Algorithm modelling. Setting the basic hyper parameters of the model.

3) Prediction on the test set directly or using forward testing.

4) Calculation of a metric that compares forecasts with expected values. Based on the evaluation of the metric, one can compare the models and accordingly select those whose accuracy satisfies the conditions of the problem.

5) Automation of work. Development of a framework for automation of one or more parts of the process.

6) According to this type of problem, the following algorithms were identified: convolutional neural network (CNN), and long short-term memory networks (LSTM). The detailed explanation of the decision of the algorithms can be found there [13].

Based on the set task and collected data were defined to use a LSTM model for the experimental prediction of the user oxygen saturation data. In Fig.5 the result of the forecasting is shown.

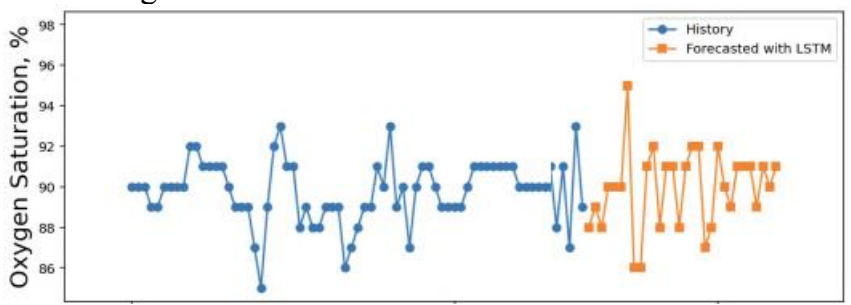

Mean Absolute Error: 2.60

Root Mean Square Error: 3.15

\section{Fig. 5. Graph with forecasted Oxygen Saturation by LSTM} model with MAE and RMSE values

Measured errors show to us that we have little difference in the forecasted and real values. They would be improved by further optimizations of the model and expanding of the dataset.
Deep Learning models have a unique processing which allows them to be more efficient than other traditional models. Models like LSTM and CNN are efficient for time series multivariate forecasting. Deep Learning models yield a high accuracy at forecasting future values. Although this is the case, even deep learning models must be used at discretion for highly dynamic datasets. Deep Learning models work as an effective modelling technique for time series forecasting.

\section{CONCLUSIONS}

Nowadays, various biomedical and healthcare tools such as genomics, mobile biometric sensors, and smartphone apps generate a big amount of data. Therefore, it is mandatory to know about and assess that can be achieved using this data. For example, the analysis of such data can provide further insights in terms of medical and other types of improvements in healthcare. It can be a preventive method of curing many illnesses.

Described steps and methods of data acquisition and processing can be applied to build more sophisticated systems for forecasting and other machine learning problems and evaluation of the created models.

Predicting the future is one of the most relevant and challenging tasks in applied sciences. Building effective predictors form historical data demands computational and statistical methods for inferring dependencies between past and short-term future values of observed values as well as appropriate strategies to deal with longer horizons.

Cyber-physical system with forecasting opportunity can be used in both clinical practice and epidemiological studies, can help monitor disease progression and in many cases prevent the growth of negative outcomes.

\section{References}

[1] Machine Learning in Wearable Biomedical Systems, August 2020, url: https://www.intechopen.com/books/sports-scienceand-human-health-different-approaches/machine-learning-inwearable-biomedical-systems

[2] Anatoliy Melnyk, Yurii Morozov, Bohdan Havano, Petro Hupalo. HealthSupervisor: Mobile Application for Roundthe-Clock Remote Monitoring of the Human Functional State (keynote). Proceedings of the 2nd International Workshop on Intelligent Information Technologies \& Systems of Information Security with CEUR-WS. Khmelnytskyi, Vol2853, Ukraine, March 24-26, 2021, pp. 24-37. - http://ceurws.org/Vol-2853/

[3] Sang M. Lee, DonHee Lee, Healthcare wearable devices: an analysis of key factors for continuous use intention, October 2020, url: https://link.springer.com/article/10.1007/s11628020-00428-3

[4] Aras R. Dargazany, Paolo Stegagno, and Kunal Mankodiya, WearableDL: Wearable Internet-of-Things and Deep Learning for Big Data Analytics - Concept, Literature, and Future, Mobile Information Systems, November 2018

[5] Using Fitness Trackers and Smartwatches to Measure Physical Activity in Research: Analysis of Consumer WristWorn Wearables, Journal of Medical Internet Research, March 2018, url: https://www.ncbi.nlm.nih.gov/pmc/articles/PMC5887043/

[6] Anatoliy Melnyk, Yuriy Morozov, Bohdan Havanio, Petro Hupalo. Investigation of Wireless Pulse Oximeters for Smartphone-based Remote Monitoring of Lung Health. Advances in Cyber-Physical Systems. 2020; Volume 5, 
$\begin{array}{lllll}\text { Number } & 2: & \text { pp. } & 70 & -\end{array}$

https://doi.org/10.23939/acps2020.02.070

[7] Ben Taieb, S., Bontempi, G., Atiya, A., Sorjamaa, A.: A review and comparison ofstrategies for multi-step ahead time series forecasting based on the NN5 forecastingcompetition. ArXiv e-prints, August 2011

[8] Jason Brownlee. Introducing to time series forecasting with Python, 2017

[9] Dash, S., Shakyawar, S.K., Sharma, M. et al. Big data in healthcare: management, analysis and future prospects. J Big Data 6, 54 (2019). https://doi.org/10.1186/s40537-019-02170 .

[10] M Supriya, AJ Deepa, Machine learning approach on healthcare big data: a review, Big Data and Information Analytics, 2020, Volume 5, Issue 1: 58-75. October 2020

[11] Aras R. Dargazany, Paolo Stegagno, and Kunal Mankodiya, WearableDL: Wearable Internet-of-Things and Deep Learning for Big Data Analytics - Concept, Literature, and Future, Mobile Information Systems, November 2018

[12] Gianluca Bontempi, Souhaib Ben Taieb, Yann-Aël Le Borgne, Machine Learning Strategies for Time Series Forecasting. Lecture Notes in Business Information Processing, January 2013, pp.138, url: https://www.researchgate.net/publication/236941795_Machi ne_Learning_Strategies_for_Time_Series_Forecasting

[13] Jason Brownlee, Deep Learning for time series forecasting, August 2018

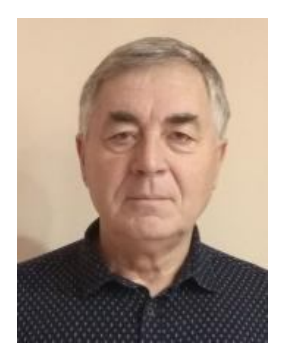

Anatoliy O. Melnyk has been a Head of the Department of Computer Engineering at Lviv Polytechnic National University since 1994 and a Head of the Department of Artificial Intelligence of John Paul II Catholic University of Lublin since 2018. He graduated from Lviv Polytechnic Institute with Engineer Degree in Computer Engineering in 1978. In 1985 he obtained his $\mathrm{PhD}$ degree in Computer Systems from Moscow Power Engineering Institute. In 1992, he received his DSc degree from the Institute of Modeling Problems in Power Engineering of the National Academy of Science of Ukraine. He was recognized for his outstanding contributions to high-performance computer systems design as a Fellow Scientific Researcher in 1988. He became a Professor of Computer Engineering in 1996. From 1982 to 1994 he was a Head of the Department of Signal Processing Systems at Lviv Radio Engineering Research Institute. From 1994 to 2008 he was a Scientific Director of the Institute of Measurement and Computer Technique at Lviv Polytechnic National University. From 1999 to 2009 he was a Dean of the Department of Computer and Information Technologies at the Institute of Business and Perspective Technologies. Since 2000 he has served as President and CEO of Intron ltd. He was also a professor at Kielce

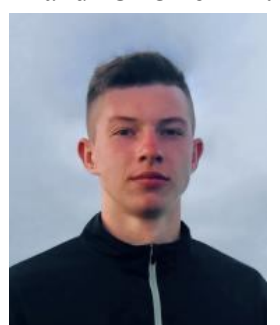
University of Technology, Rzeszow University of Information Technology and Management, a visiting professor at University of Bielsko-Biala.

Petro Hupalo is a fourth-year student in computer engineering at Lviv Polytechnic National University. He was involved in some technological startups. His interests are related to the development of software solutions, machine learning and data analytics 